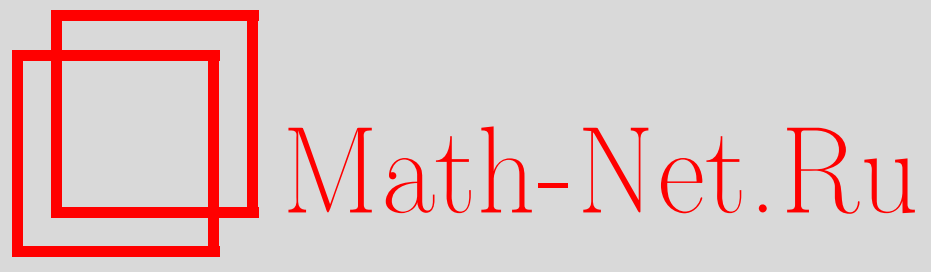

А. Э. Дружинин, О гомотопически инвариантных предпучках с Witt-трансферами, УМH, 2014, том 69, выпуск $3,181-182$

DOI: https://doi.org/10.4213/rm9593

Использование Общероссийского математического портала Math-Net.Ru подразумевает, что вы прочитали и согласны с пользовательским соглашением http://www . mathnet.ru/rus/agreement

Параметры загрузки:

IP : 34.227 .88 .159

26 апреля 2023 г., 13:15:06

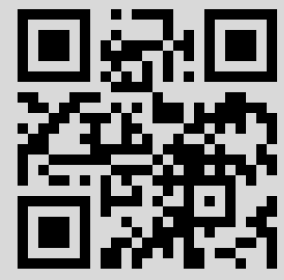




\section{О гомотопически инвариантных предпучках с Witt-трансферами}

\section{А. Э. Дружинин}

В. А. Воеводский в [1] построил триангулированную категорию мотивов $\mathrm{DM}^{-}(k)$, И. А. Панин поставил задачу построения триангулированной категории Witt-мотивов. Первым шагом в построении этой категории является доказательство теоремы 3 ниже.

Пусть $k$ - поле характеристики, не равной 2. Пусть $\mathrm{Sm}_{k}$ - категория аффинных гладких многообразий над полем $k$. Определим аддитивную категорию Wor $_{k}$.

ОПредЕлЕниЕ 1. Объекты категории $\mathrm{Wor}_{k}$ совпадают с объектами $\mathrm{Sm}_{k}$; морфизмы $\operatorname{Wor}_{k}(X, Y)$ - группа Витта категории $k[X \times Y]$-модулей, конечно порожденных и проективных над $k[X]$, с двойственностью $P \mapsto \widehat{P_{k[X]}}=\operatorname{Hom}_{k[X]}(P, k[X])$ (в соответствии с определением 27 из [2]); композиция морфизмов $\Phi \in \operatorname{Wor}_{k}(X, Y)$ и $\Psi \in$ Wor $_{k}(Y, Z)$ определяется как тензорное произведение над $k[Y]$ соответствующих квадратичных пространств.

ЗАмечание 1. (i) Определен функтор $\mathrm{Sm}_{k} \rightarrow$ Wor $_{k}$. Этот функтор на объектах действует тождественно, а регулярное отображение $f: X \rightarrow Y$ переводит в морфизм, определяемый бимодулем $k_{k[X]} k[X]_{k[Y]}$ и каноническим изоморфизмом $k[X]_{k[Y]} \simeq$ $\operatorname{Hom}_{k[X]}\left(k[X]_{k[Y]}, k[X]\right)$.

(ii) Категорию Wor $_{k}$ можно расширить на пары $\left(X_{1}, X_{2}\right)$, где $X_{2}$ - открытое подмножество $X_{1}$, определив морфизмы между парами $i_{X}: X_{2} \hookrightarrow X_{1}, i_{Y}: Y_{2} \hookrightarrow Y_{1}$ :

$$
\begin{aligned}
\operatorname{Wor}_{k} & \left(\left(X_{1}, X_{2}\right),\left(Y_{1}, Y_{2}\right)\right) \\
\quad= & \operatorname{coker}\left(\operatorname{Wor}_{k}\left(X_{1}, Y_{2}\right) \stackrel{\left(i_{Y} \circ-,-\circ i_{X}\right)}{\longrightarrow} \operatorname{Wor}_{k} \cdot{ }^{\cdot}\left(X_{2} \rightarrow X_{1}, Y_{2} \rightarrow Y_{1}\right)\right),
\end{aligned}
$$

где $\operatorname{Wor}_{k}^{\cdot} \rightarrow \cdot$ - категория стрелок.

Определение 2. Объекты категории $\overline{\mathrm{Wor}_{k}}$ совпадают с объектами $\mathrm{Wor}_{k}$; морфизмы определяются так: $\overline{\operatorname{Wor}_{k}}(X, Y)=\operatorname{coker}\left(\operatorname{Wor}_{k}\left(\mathbb{A}^{1} \times X, Y\right) \stackrel{\left(-\circ i_{0}\right)-\left(-\circ i_{1}\right)}{\longrightarrow} \operatorname{Wor}_{k}(X, Y)\right)$, где $i_{0}, i_{1}: X \hookrightarrow \mathbb{A}^{1} \times X$ - нулевое и единичное сечения $\mathbb{A}^{1} \times X$.

ОПредЕлЕниЕ 3. Предпучок абелевых групп с Witt-трансферами - это произвольный предпучок $F:$ Wor $_{k} \rightarrow \mathrm{Ab}$ абелевых групп на категории $\mathrm{Wor}_{k}$.

ЗАмечАние 2. Предпучок с Witt-трансферами называется гомотопически инвариантным, если он гомотопически инвариантен после ограничения на $\mathrm{Sm}_{k}$. Такой предпучок пропускается через $\mathrm{Wor}_{k} \rightarrow \overline{\text { Wor }_{k}}$. Обратно, всякий предпучок на Wor $_{k} \rightarrow \overline{\text { Wor }_{k}}$ гомотопически инвариантен.

Теорема 1. Пусть $\mathscr{F}$ - гомотопически инвариантный предпучок с Witt-трансферами, тогда для двух вложенных окрестностей по Зарисскому $U \subset V$ точки z в $\mathbb{A}_{K}^{1}($ әде $K=k(X)$ - поле частных многообразия $X)$ ограничение

$$
i^{*}: \frac{\mathscr{F}(V-z)}{\mathscr{F}(V)} \rightarrow \frac{\mathscr{F}(U-z)}{\mathscr{F}(U)}
$$

является изоморфизмом ( $і$ обозначает вложение $U$ в $V$ ).

Доказательство теоремы 1 выводится из следующей леммы.

Лемма 1. Морфизм включения $[i]:(U, U-z) \rightarrow(V, V-z)$ в $\overline{\text { Wог }_{k}}$ является изоморфизмом.

Работа выполнена при поддержке РФФИ (грант № 14-01-31095).

DOI: $10.4213 / \mathrm{rm} 9593$ 
Теорема 2. Пусть Я્F - гомотопически инвариантный предпучок $c$ Witt-трансферами и $\pi: X^{\prime} \rightarrow X$ - этальный морфизм гладких кривых над полем $K$, являющимся полем частных некоторого гладкого афбинного многообразия. Пусть $z \in X-$ замкнутая точка такая, что $\pi^{-1}(z)$ состоит из одной точки, скажем, $z^{\prime}$, и индуцированный на полях вычетов этих двух точек гомоморфизм является изоморфизмом. Тогда $\pi$ индуиирует изоморфизм

$$
\pi^{*}: \frac{\mathscr{F}(U-z)}{\mathscr{F}(U)} \stackrel{\sim}{\longrightarrow} \frac{\mathscr{F}\left(U^{\prime}-z^{\prime}\right)}{\mathscr{F}\left(U^{\prime}\right)}, \quad \text { əде } \quad U=\operatorname{Spec}\left(\mathcal{O}_{X, z}\right), \quad U^{\prime}=\operatorname{Spec}\left(\mathcal{O}_{X^{\prime}, z^{\prime}}\right) .
$$

Доказательство теоремы 2 выводится из следующей леммы.

Лемма 2. Пусть $\pi: X \rightarrow X^{\prime}$ определен в теореме 2 , и пусть канонические классы $X$ и $X^{\prime}$ тривиальны, пусть $U=\operatorname{Spec}\left(\mathcal{O}_{X, z}\right), U^{\prime}=\operatorname{Spec}\left(\mathcal{O}_{X^{\prime}, z^{\prime}}\right)$, а $i$ и $i^{\prime}$ - вложения $U$ и $U^{\prime}$ в $X$ и $X^{\prime}$ соответственно. Тогда:

(a) существует $\Phi \in \operatorname{Wor}_{K}\left((U, U-z),\left(X^{\prime}, X^{\prime}-z^{\prime}\right)\right)$ такой, что $[\pi \circ \Phi]=[i]$ в $\overline{\mathrm{Wor}_{K}}((U, U-z),(X, X-z))$;

(b) существует $\Psi \in \operatorname{Wor}_{K}\left((U, U-z),\left(X^{\prime}, X^{\prime}-z^{\prime}\right)\right)$ такой, что $[\Psi \circ \pi]=\left[i^{\prime}\right]$ в $\overline{\operatorname{Wor}_{K}}\left(\left(U^{\prime}, U^{\prime}-z^{\prime}\right),\left(X^{\prime}, X^{\prime}-z^{\prime}\right)\right)$.

Теорема 3. Для гомотопически инвариантного предпучка $\mathscr{F}$ с Witt-трансфера-

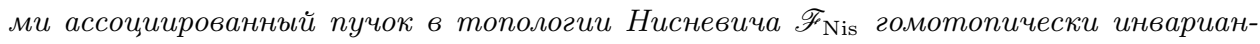
тен.

Доказательство теоремы 3 выводится из двух лемм (доказанных в [3] и [4]).

Лемма 3. Пусть $\mathscr{F}$ - гомотопически инвариантный пучок с Witt-трансферами. Тогда пучки $\mathscr{F}_{\text {Zar }}$ и $\mathscr{F}_{\text {Nis }}$ совпадают при ограничении на $\mathbb{A}_{K}^{1}$ для произвольного поля $K$, являющегося полем частных некоторого гладкого многообразия.

Лемма 4. Пусть Я્F-гомотопически инвариантный пучок с Witt-трансферами. Тогда каноническое отображение $\mathscr{F}(U) \rightarrow \mathscr{F}_{\mathrm{Zar}}(U)$ сюрвективно для любого открытого по Зарисскому подмножества $U \subset \mathbb{A}_{K}^{1}$, где $K$ - произвольное поле, являющееся полем частных некоторого гладкого многообразия.

Автор благодарен И. А. Панину за постановку задачи.

\section{Список литературы}

[1] V. Voevodsky, "Triangulated category of motives over a field", Cycles, transfers, and motivic homology theories, Ann. of Math. Stud., 143, eds. V. Voevodsky, A. Suslin, E. Friedlander, Princeton Univ. Press, Princeton, NJ, 2000, 188-238. [2] P. Balmer, "Witt groups", Handbook of K-theory, v. 2, eds. E. M. Friedlander, D. R. Grayson, Springer, Berlin, 2005, 539-576. [3] А.Э. Дружинин, Сохранение гомотопической инвариантности предпучков с Witt-трансферами при пучковании в топологии Зарисского, Препринт 07/2014, ПОМИ, СПб., 2014, 10 с., http://www.pdmi.ras.ru/preprint/2014/14-07.html. [4] А. Дружинин, Сохранение гомотопической инвариантности предпучков с Witt-трансферами при пучковании в топологии Нисневича, Препринт 8/2014, ПОМИ, СПб., 2014, 14 с., http://www.pdmi.ras.ru/preprint/2014/14-08.html.

Андрей Эдуардович Дружинин

(Andrei È. Druzhinin)

Санкт-Петербургский государственный университет

E-mail: andrei.druzh@gmail.com
Представлено Д. О. Орловым Принято редколлегией 22.05 .2014 